\title{
Radial-velocity jitter of stars as a function of observational timescale and stellar age ${ }^{\star, \star \star}$
}

\author{
Stefan S. Brems ${ }^{1}$, Martin Kürster ${ }^{2}$, Trifon Trifonov ${ }^{2}$, Sabine Reffert ${ }^{1}$, and Andreas Quirrenbach ${ }^{1}$ \\ 1 Zentrum für Astronomie der Universität Heidelberg, Landessternwarte, Königstuhl 12, 69117 Heidelberg, Germany \\ e-mail: sbrems@lsw.uni-heidelberg.de \\ 2 Max Planck Institut für Astronomie, Königstuhl 17, 69117 Heidelberg, Germany
}

Received 22 March 2019 / Accepted 8 October 2019

\begin{abstract}
Context. Stars show various amounts of radial-velocity (RV) jitter due to varying stellar activity levels. The typical amount of RV jitter as a function of stellar age and observational timescale has not yet been systematically quantified, although it is often larger than the instrumental precision of modern high-resolution spectrographs used for Doppler planet detection and characterization.

Aims. We aim to empirically determine the intrinsic stellar RV variation for mostly $\mathrm{G}$ and $\mathrm{K}$ dwarf stars on different timescales and for different stellar ages independently of stellar models. We also focus on young stars ( $\lesssim 30 \mathrm{Myr}$ ), where the RV variation is known to be large.

Methods. We use archival FEROS and HARPS RV data of stars which were observed at least 30 times spread over at least two years. We then apply the pooled variance (PV) technique to these data sets to identify the periods and amplitudes of underlying, quasiperiodic signals. We show that the PV is a powerful tool to identify quasiperiodic signals in highly irregularly sampled data sets.

Results. We derive activity-lag functions for 20 putative single stars, where lag is the timescale on which the stellar jitter is measured. Since the ages of all stars are known, we also use this to formulate an activity-age-lag relation which can be used to predict the expected RV jitter of a star given its age and the timescale to be probed. The maximum RV jitter on timescales of decades decreases from over $500 \mathrm{~m} \mathrm{~s}^{-1}$ for $5 \mathrm{Myr}$-old stars to $2.3 \mathrm{~m} \mathrm{~s}^{-1}$ for stars with ages of around $5 \mathrm{Gyr}$. The decrease in RV jitter when considering a timescale of only $1 \mathrm{~d}$ instead of $1 \mathrm{yr}$ is smaller by roughly a factor of 4 for stars with an age of about $5 \mathrm{Myr}$, and a factor of 1.5 for stars with an age of $5 \mathrm{Gyr}$. The rate at which the RV jitter increases with lag strongly depends on stellar age and reaches $99 \%$ of the maximum RV jitter over a timescale of a few days for stars that are a few million years old, up to presumably decades or longer for stars with an age of a few gigayears.
\end{abstract}

Key words. stars: activity - methods: data analysis - techniques: radial velocities

\section{Introduction}

Of the almost 4000 exoplanets known today, more than 3700 were discovered via the radial-velocity (RV) or transit methods ${ }^{1}$. According to the database, only three of them (V830 Tau b, Donati et al. 2016; K2-33 b, David et al. 2016, and TAP $26 \mathrm{~b}$, Yu et al. 2017) are younger than $100 \mathrm{Myr}$. The main reason for this is the strong stellar activity of young stars, which makes it hard to find the subtle planetary signal in the large stellar variations. This is unfortunate for two reasons: first, planet formation takes place in young systems and at least gas giants need to form before the disk has dissipated after less than a few tens of millions of years (e.g., Ercolano \& Pascucci 2017). Second, planets at large orbital distances $(\gtrsim 50 \mathrm{AU})$ are almost exclusively detected via direct imaging (DI), which is best applicable to young systems where the planets are still hot from their formation. Thus, in order to discover all planets in a system, one either needs to image old stars - which seems currently impossible given the already low detection rate around young stars probed

* Table E.1 is only available at the CDS via anonymous ftp to cdsarc.u-strasbg.fr $(130.79 .128 .5)$ or via http://cdsarc. u-strasbg.fr/viz-bin/cat/J/A+A/632/A37

$\star \star$ Based on data obtained from the ESO Science Archive Facility under request number sbrems392771.

1 https://exoplanetarchive.ipac.caltech.edu by large DI surveys (e.g., Desidera et al. 2015; Lannier et al. 2016; Tamura 2016; Stone et al. 2018) - or try to minimize the impact of the stellar activity of young stars. A lot has been done to understand and characterize stellar activity (e.g., Dumusque 2018). Lindegren \& Dravins (2003) further estimate the effects of stellar activity such as oscillation, granulation, meridional flow, long-term magnetic cycle, surface magnetic activity and rotation, gravitational redshift and many more on the RV measurement. Meunier \& Lagrange (2019) then try to model the effect of this kind of activity signal on RV data of mature stars. With our data probing activity timescales of days to years, we are mainly probing the combined effect of stellar rotation, reconfiguration of active regions, and long-term magnetic cycles. Still, large uncertainties remain in the prediction and interpretation of any RV signal, in particular for young pre-main sequence stars. But since this is what we measure, knowledge about the typical RV variability is important, for example for developing and testing RV activity models or planning RV surveys. In this paper we therefore derive a model-free analytic relation between stellar jitter, stellar age, and lag, where lag denotes the timescale on which the jitter is measured.

In order to derive this relation, we systematically analyze precise Doppler measurements from FEROS (Kaufer et al. 1999) and HARPS (Mayor et al. 2003) of mainly $\mathrm{G}$ and $\mathrm{K}$ dwarfs with ages between $3 \mathrm{Myr}$ and $7 \mathrm{Gyr}$ using the pooled variance (PV) technique (Donahue et al. 1997a,b; Kürster et al. 2004). 
This paper is organized as follows: in Sect. 2 we introduce the input target list as well as target selection and data cleaning. Section 3 explains the PV technique, the activity modeling for individual stars, and the uncertainty estimation. Section 4 presents two analytic activity-age-lag functions to the pooled data of all stars. In Sect. 5 we discuss the strengths, weaknesses, and limits of this analysis and give example values of the empirical activity-age-lag function. Section 6 then concludes on the main findings of this paper.

\section{Target sample}

Our goal is to characterize RV jitter as a function of stellar age and probed timescale. Therefore, we need to put together a sample of young and old stars with known ages that have been part of RV monitoring programs.

We assemble our input target list from three different surveys which all focus on young stars in the southern hemisphere: first, the ongoing Imaging Survey for Planets around Young stars (NaCo-ISPY) ${ }^{2}$ survey (Launhardt et al., in prep.; 443 targets). Second the recently started Radial-velocity Survey for Planets around Young stars (RV SPY) survey (Zakhozhay et al., in prep.; 180 targets). And third the RV survey to find planets around young stars from Weise (2010) and Mohler-Fischer (2013; 214 targets). NaCo-ISPY is a 120-night guaranteed time observations (GTO) $L^{\prime}$-band direct imaging survey using the NaCo instrument (Lenzen et al. 2003; Rousset et al. 2003) at the VLT. Its target list contains mostly two subcategories: first, young, nearby stars with debris disks showing significant infrared excess, and second, stars with protoplanetary disks. RVSPY is a RV survey currently spanning 40 nights that is complementary to NaCo-ISPY, as it searches for close-in planets around debris disk stars older than about $10 \mathrm{Myr}$. Its target list has an intentionally large overlap with the NaCo-ISPY target list, but is extended especially for older, less active stars. Since the second and third surveys use the FEROS instrument and young stars are mostly avoided in RV surveys, the young stars ( $\leq 45 \mathrm{Myr})$ are uniquely observed with FEROS; see Table 1. However, since their intrinsic RV variation is at least one order of magnitude larger than the instrumental precision, we do not expect any significant bias in our results from this selection effect. Since there are overlaps in the catalogs, we end up with 699 targets. Figure 1 shows the age distribution of those stars. One can see that most stars in the input catalog are younger than $100 \mathrm{Myr}$. Almost all of the older stars come from the RV SPY survey, which also included older stars to avoid the issues of young and active stars as RV targets.

We searched the archives for public data from the ESOinstruments HARPS (Mayor et al. 2003) and FEROS (Kaufer et al. 1999) for all 699 stars in our input catalog. The RVs were derived using the CERES pipeline (Brahm et al. 2017) for FEROS and the SERVAL pipeline (Zechmeister et al. 2018) for HARPS data. We removed bad observations with formal errors as returned by the pipelines above $20 \mathrm{~m} \mathrm{~s}^{-1}$ and $50 \mathrm{~m} \mathrm{~s}^{-1}$ for HARPS and FEROS respectively, and via iterative $10 \sigma-$ clipping on all data of a star simultaneously. In order to qualify for our final analysis, the remaining data need to be sufficiently evenly distributed for each star and instrument. We ensured this by requiring a minimum of 30 observations, at least a two-year baseline, no gap larger than $50 \%$, and a maximum of two gaps larger than $20 \%$ of the baseline. Since HARPS underwent a major intervention, including a fiber change in June 2015

2 http://wWW.mpia.de/NACO_ESPRI_GTO
(Lo Curto et al. 2015), these criteria needed to be fulfilled for one of the data sets before or after the intervention.

Finally stars with known companions (stellar or substellar) listed in the Washington Double Star catalog (Mason et al. 2001), the Spectroscopic Binary Catalog (9th edition Pourbaix et al. 2004), or the NASA Exoplanet Archive ${ }^{1}$ were also removed. This is done so we can make the assumption that we are left only with stellar noise. As a consistency check with increased statistics, we additionally analyzed seven of the removed wide binary systems ( $\geq 27$ AU projected separation). They are not used to obtain the main results given in Table 2 and are marked with a binary flag in Tables 1 and 2 .

After this selection we were left with 27 stars (including the 7 binaries): 9 with sufficient HARPS data and 19 with sufficient FEROS data, where HD 25457 (single) had good data from both instruments. Table 2 lists their basic properties and Fig. C.1 shows the RV data for all 27 stars. The individual measurements, including the barycentric Julian date (BJD), the RV signal, and the RV error, are given in Table E.1. These data are only available at the CDS.

\section{Method}

\subsection{Models}

In order to determine the typical RV scatter over different observing timescales (lags), several methods were applied.

First, the variogram or structure function (Hughes et al. 1992) using different estimators as described in Rousseeuw \& Croux (1993) and Eyer \& Genton (1999) was tried: the idea here is to create all possible differences between the measured RV data points. Those points are then sorted by the time differences (lags) and compared to theoretical predictions of a sinusoidal signal. Second, self-created, automated block-finding algorithms with random selection of single observation in clustered observations: The algorithm identifies clustered observations on different timescales. For clusters shorter than an arbitrarily chosen fraction of the lag probed, one random observation will be picked, while longer blocks will be split into sub-blocks of the according size. For each block, the variance is determined and used as the typical variance for that timescale. Third, consecutive binning was tried, where the observations are binned on the timescale chosen. This is similar to the second method, but there is no upper limit on the block size and one takes the mean instead of a random representative of that bin. This is done for all lags to be probed. And finally the PV was used.

Since only the latter method turned out to be robust enough to identify signals in sparse and irregularly sampled data, we used it for our analysis. The remainder of this section is dedicated to describing the method in more detail.

\subsection{Pooled variance}

We use the PV or pooled variance diagram (PVD) method, which was first introduced for the analysis of time series of astronomical data by Dobson et al. (1990) to analyze the CaII emission strength of active late-type stars. Dobson et al. (1990), Donahue et al. (1995, 1997a,b), Donahue \& Dobson (1996), and Kürster et al. (2004) demonstrated the capability of this technique to detect timescales pertaining to stellar activity such as the stellar rotation period, the typical timescale of active region reconfiguration or the stellar activity cycle. The PV is a combined variance estimate from $k$ different sets of measurements $y_{i, j}, i=1, \ldots, N_{j}$, $j=1, \ldots, k$ each of which has a different mean $\overline{y_{j}}$. If it can be 
Table 1. Properties of the 27 stars that qualified for further analysis.

\begin{tabular}{|c|c|c|c|c|c|c|c|c|}
\hline Main ID & RA & Dec & SpT & $\begin{array}{c}\text { Age } \\
{[\mathrm{Myr}]}\end{array}$ & Instrument & Catalog & Age ref. & $\begin{array}{c}\text { Bin. Sep. } \\
{[\mathrm{AU}]}\end{array}$ \\
\hline V2129 Oph & $16: 27: 40.286$ & $-24: 22: 04.030$ & K7 & $3 \pm 2$ & FEROS & 1,2 & 4 & 78 \\
\hline HD 140637 & $15: 45: 47.600$ & $-30: 20: 56.000$ & $\mathrm{~K} 2 \mathrm{~V}$ & $5 \pm 2$ & FEROS & 2 & 5 & 27 \\
\hline CD-37 13029 & 19:02:02.000 & $-37: 07: 44.000$ & G5 & $5 \pm 2$ & FEROS & 2 & 5 & \\
\hline TYC 8654-1115-1 & $12: 39: 38.000$ & $-57: 31: 41.000$ & G9V & $5 \pm 3$ & FEROS & 2 & 4 & \\
\hline HBC 603 & $15: 51: 47.000$ & $-35: 56: 43.000$ & M0 & $5 \pm 3$ & FEROS & 2 & 4 & 280 \\
\hline HD 81544 & $09: 23: 35.000$ & $-61: 11: 36.000$ & $\mathrm{~K} 1 \mathrm{~V}$ & $8 \pm 3$ & FEROS & 2 & 4 & 591 \\
\hline TYC 5891-69-1 & $04: 32: 43.509$ & $-15: 20: 11.268$ & G4V & $10 \pm 5$ & FEROS & 2 & 4 & \\
\hline CD-78 24 & $00: 42: 20.300$ & $-77: 47: 40.000$ & $\mathrm{~K} 3 \mathrm{~V}$ & $15 \pm 10$ & FEROS & 2 & 4 & \\
\hline 1RXS J043451.0-354715 & $04: 34: 50.800$ & $-35: 47: 21.000$ & $\mathrm{~K} 1 \mathrm{~V}$ & $20 \pm 15$ & FEROS & 2 & 4 & \\
\hline 1RXS J033149.8-633155 & $03: 31: 48.900$ & $-63: 31: 54.000$ & KOV & $25 \pm 15$ & FEROS & 2 & 4 & \\
\hline TYC 7697-2254-1 & 09:47:19.900 & $-40: 03: 10.000$ & $\mathrm{~K} 0 \mathrm{~V}$ & $25 \pm 10$ & FEROS & 2 & 4 & \\
\hline CD-37 1123 & 03:00:46.900 & $-37: 08: 02.000$ & G9V & $30 \pm 15$ & FEROS & 2 & 4 & \\
\hline CD-84 80 & $07: 30: 59.500$ & $-84: 19: 28.000$ & G9V & $30 \pm 15$ & FEROS & 2 & 4 & \\
\hline HD 51797 & $06: 56: 23.500$ & $-46: 46: 55.000$ & $\mathrm{~K} 0 \mathrm{~V}$ & $30 \pm 15$ & FEROS & 2 & 4 & \\
\hline TYC 9034-968-1 & $15: 33: 27.500$ & $-66: 51: 25.000$ & $\mathrm{~K} 2 \mathrm{~V}$ & $30 \pm 15$ & FEROS & 2 & 4 & \\
\hline HD 30495 & $04: 47: 36.210$ & $-16: 56: 05.520$ & $\mathrm{G} 1.5 \mathrm{~V}$ & $45 \pm 10$ & HARPS & 1,3 & 6 & \\
\hline HD 25457 & $04: 02: 36.660$ & $-00: 16: 05.920$ & F7V & $50 \pm 15$ & Both & $1,2,3$ & 4 & \\
\hline 1RXS J223929.1-520525 & $22: 39: 30.300$ & $-52: 05: 17.000$ & $\mathrm{~K} 0 \mathrm{~V}$ & $60 \pm 15$ & FEROS & 2 & 4 & \\
\hline HD 96064 & $11: 04: 41.580$ & $-04: 13: 15.010$ & G5 & $90 \pm 10$ & FEROS & 2 & 4 & 123 \\
\hline HD 51062 & $06: 53: 47.400$ & $-43: 06: 51.000$ & G5V & $200 \pm 50$ & FEROS & 2 & 4 & SB \\
\hline HD 202628 & $21: 18: 27.269$ & $-43: 20: 04.750$ & G5V & $604 \pm 445$ & HARPS & 1,3 & 7 & \\
\hline HD 191849 & $20: 13: 52.750$ & $-45: 09: 49.080$ & MOV & $850 \pm 400$ & HARPS & 1,3 & 8 & \\
\hline HD 199260 & $20: 56: 47.331$ & $-26: 17: 46.960$ & F6V & 3460 & HARPS & 1,3 & 9 & \\
\hline HD 1581 & 00:20:01.910 & $-64: 52: 39.440$ & F9V & 3950 & HARPS & 1 & 8 & \\
\hline HD 45184 & $06: 24: 43.880$ & $-28: 46: 48.420$ & $\mathrm{G} 2 \mathrm{~V}$ & 4420 & HARPS & 3 & 10 & \\
\hline HD 154577 & $17: 10: 10.270$ & $-60: 43: 48.740$ & KOV & 4800 & HARPS & 3 & 10 & \\
\hline HD 43834 & 06:10:14.200 & $-74: 45: 09.100$ & G5V & $7244 \pm 3226$ & HARPS & 1 & 11 & 32 \\
\hline
\end{tabular}

Notes. Column "Bin. Sep." lists the projected separation of the binary companion, if present, to the host star as listed in the Washington Double Star catalog (WDS). The acronym SB refers to a spectroscopic binary identified in our data.

References. (1) ISPY Launhardt et al. (in prep.); (2) Mohler-Fischer (2013) and Weise et al. (2010); (3) RV SPY Zakhozhay et al. (in prep.); (4) Weise et al. (2010); (5) Weise (2010); (6) Maldonado et al. (2010); (7) Tucci Maia et al. (2016); (8) Vican (2012); (9) Ibukiyama \& Arimoto (2002); (10) Chen et al. (2014); (11) Lachaume et al. (1999).

assumed that all the individual sets of measurements have the same variance (despite the different mean), then the PV $\sigma_{\mathrm{p}}$ is defined as

$$
\begin{aligned}
\sigma_{\mathrm{p}, k}^{2} & :=\frac{\left(N_{1}-1\right) \sigma_{1}^{2}+\ldots+\left(N_{k}-1\right) \sigma_{k}^{2}}{\left(N_{1}-1\right)+\ldots+\left(N_{k}-1\right)} \\
& =\frac{\sum_{i=1}^{N_{1}}\left(y_{i, 1}-\overline{y_{1}}\right)^{2}+\ldots+\sum_{i=1}^{N_{k}}\left(y_{i, k}-\overline{y_{k}}\right)^{2}}{N_{1}+\ldots+N_{k}-k},
\end{aligned}
$$

with $\sigma_{\mathrm{p}, j}$ being the variance of the $j$ th data set, that is, the PV is the weighted mean of the variances of the individual data sets.

In this paper we make the assumption of (on average) equal $\mathrm{RV}$ variances in spite of different mean RV for data sets that were obtained within time intervals of equal length. In particular we assume this to be true for the data taken by HARPS before and after the intervention in June 2015 (Lo Curto et al. 2015), even though the formal errors increase from a mean of $0.9 \mathrm{~m} \mathrm{~s}^{-1}$ to $1.3 \mathrm{~m} \mathrm{~s}^{-1}$ for our data. This decreased precision after the intervention seems to affect all HARPS data (Trifonov, in prep.), but since we probe jitter values typically much larger than these formal errors, the assumption still holds. The only significant effect is the offset of several meters per second in the absolute RV values. To account for this, we split the data into pre- and post-data sets. Furthermore, we assume that the RV variance on a given timescale does not change over time (e.g., due to activity cycles). For these data sets the PV can be calculated and is used as an estimate of the true variance for this timescale. As long as the lag probed is shorter than the observational baseline, the PV is more precise than the variance of a single data set due to the larger number of measurements as several data sets are combined. If the length of the time intervals differs we expect different variances in general.

\subsection{Block sizes}

As the integer $k$ to split our observations in smaller blocks is arbitrary, we run $k=1, \ldots, B$, where $B$ is the number of days covered by the observations. In other words, we split our data into blocks, starting from one block having the full baseline length, to $B$ blocks with a length of one day each. We define the lag $\tau:=B / k$, which corresponds to the length of each bin in days for a given $k$. For each value of $k$, or equivalent lag $\tau$, we then obtain a different variance $\sigma_{\mathrm{p}, k}^{2}$ as defined in Eq. (1). We note that due to the HARPS fiber change in June 2015 (Lo Curto et al. 2015), we removed HARPS data taken during this procedure (2457173.02457177.0 JD) and did not allow blocks to combine data taken before and after the intervention. We therefore had two data sets with shortened baselines. After applying the PV, we then treat the two datasets as one again. 


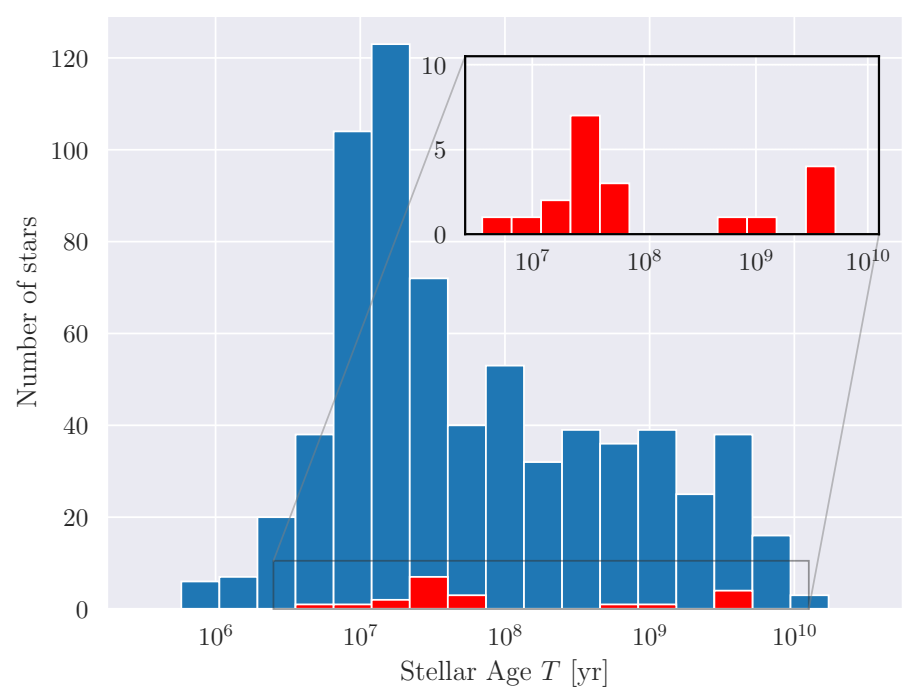

Fig. 1. Age distribution of the stars in the input catalog (blue) and the remaining 20 single stars (red) after applying the selection criteria described in the text. The remaining sample has a wide age distribution, slightly skewed towards younger stars. Young stars $(\leq 100 \mathrm{Myr})$ are especially important for this analysis and are in general rarely observed in RV surveys.

It is difficult to assign absolute error bars to each pooled data point, as the formal RV precision (a few $\mathrm{m} \mathrm{s}^{-1}$ ) is typically much smaller than the PV (a few hundred $\mathrm{m} \mathrm{s}^{-1}$ ). We therefore decided to assign a weight $w_{k}$ to each measurement $\sigma_{\mathrm{p}, k}$. In contrast to errors, weights only have a relative meaning and thus do not need to be calibrated absolutely. Since more points yield in general a more significant result, we use the square root of the number of individual points minus the number of filled boxes that were used to compute this point. We subtract the number of filled boxes because each box takes one degree of freedom (the mean):

$w_{k}:=\sqrt{\frac{n_{k}-\tilde{k}}{2}}$,

where $n_{k}:=N_{1}+\ldots+N_{\tilde{k}}$ is the number of individual data points contributing, and $\tilde{k}$ is the number of blocks where the variance could be calculated, that is, the number of boxes with at least two measurements. This formula, and especially the subtraction of $\tilde{k}$, are further motivated by the relative uncertainty of the variance estimator for $N$ points, which is $\sqrt{\frac{2}{N-1}}$ (e.g., Squires 2001, p. 22). Thus, under the assumption of $k$ independent blocks with $N / k$ measurements in each block, the uncertainty is

$\sqrt{\frac{2}{N / k-1}} \cdot \frac{1}{\sqrt{k}}=\sqrt{\frac{2}{N-k}} \equiv w_{k}^{-1}$

for $k$ independent blocks with $N / k$ measurements in each block (compare also Brown \& Levine 2007).

\subsection{Activity modeling}

Now that we know the variance on different timescales, we want to find the periods $P$ and amplitudes $K$ of the underlying modulations. Under the assumption of an underlying, infinitely sampled sinusoidal signal $y(t)=K \sin \left(\frac{2 \pi}{P} t-\delta\right)$ with semi-amplitude $K$, period $P$, and phase $\delta$, the analytic PV for timescale $\tau$ is given in Eq. (B.4), which is independent of $\delta$.
Since there might be no, one, or multiple periodic signals of this kind, we fit four different curves to each PV data point of a star:

$\sigma_{\mathrm{p}}^{2}(\tau)=A^{2}+\sum_{i=1}^{m} K_{i}^{2}\left[\frac{1}{2}+\frac{\cos \left(2 \pi \tau / P_{i}\right)-1}{\left(2 \pi \tau / P_{i}\right)^{2}}\right]$,

where $m \in[0,1,2,3]$ is the number of sinusoidal signals and $\tau$ is the timescale or lag probed. In other words, only white noise or white noise plus up to three underlying sinusoidal signals are fitted using $\chi^{2}$-minimization, where the formal squared errors are given by the reciprocal of the weight from Eq. (2). We note that the models have $2 m+1$ free parameters. In order to decide how many signals are significant, we used an F-test (Rawlings et al. 1998), which is often used in nested models. We highlight the fact that the number of independent measurements required in this test is the number of observations and not the number of points in the PVD. This approach has one value $\alpha$ to be chosen arbitrarily. This $\alpha$ acts as a threshold in rejecting the null hypothesis, which is that the model with fewer parameters describes the data as well as the model with more parameters. It is typically chosen to be around $\alpha \approx 0.01-0.05$, where a larger value favors the model with fewer parameters. We select $\alpha=0.05$.

\subsection{Uncertainty estimation}

Finally we want to assign confidence intervals to each of the $2 m+1$ parameters we found for each star to best describe its activity. We tried the following methods:

First, we tried to apply Markov chain Monte Carlo (MCMC) on the RV data as well as on the pooled data. Then we tried a Monte Carlo (MC)-like method on the pooled data: we removed the modeled signal from the pooled data, binned seven neighboring data points, for example, and determined their mean and variance. This mean and variance were then used to create seven new, random data points, assuming a Gaussian distribution. We then re-added the modeled signal and re-performed the fitting routine. And finally we tried bootstrap resampling on the RV data as well as on the pooled data.

Quantifying these methods not only by eye but also using artificially generated data where we know the true periods and amplitudes of the underlying signals, we found the last method to give the most realistic results. By "realistic", we mean that the true value lies within the error bars of the estimated values and that the sampled values spread roughly equally around the original estimate. The MCMC methods underestimated the errors, whereas in the MC-like method one often has the problem of non-Gaussian distributed residuals, which will then lead to skewed and shifted results if those are approximated and redistributed by Gaussians. Bootstrapping the original data leads to issues when bins often contain identical data, returning a variance of zero, seemingly skewing the results to smaller absolute variances.

For the last method, we pooled the data using Eq. (1) as described in Sects. 3.2 and 3.3, resulting in $k$ pairs of lag $\tau$ and variance $\sigma_{\mathrm{p}}^{2}$. Subsequently, we determined the model capable of best describing the data using the F-test; see Sect. 3.4. We randomly redrew $k$ pairs of lag $\tau$ and variance $\sigma_{\mathrm{p}}^{2}$ with placing back, meaning the same pair can be drawn multiple times. This is the method known as bootstrapping. Fixing the model to the one found for the original data, we fit this model to the new data. Repeating the last two steps 5000 times yields an estimate of the robustness of the model parameters. The original fit is used as the best fit and the standard deviations left and right of that 
S. S. Brems et al.: Radial-velocity jitter of stars as a function of observational timescale and stellar age

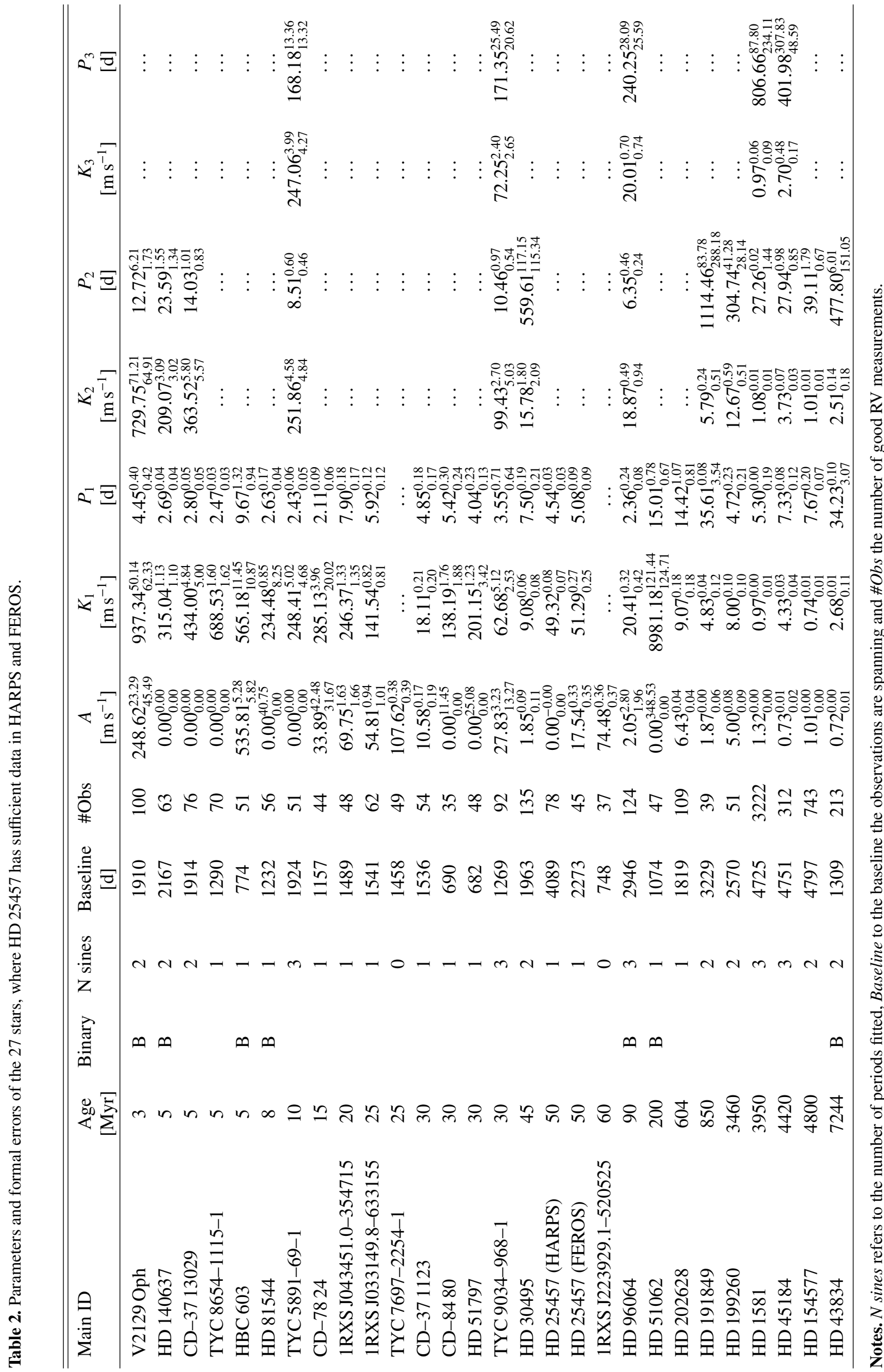



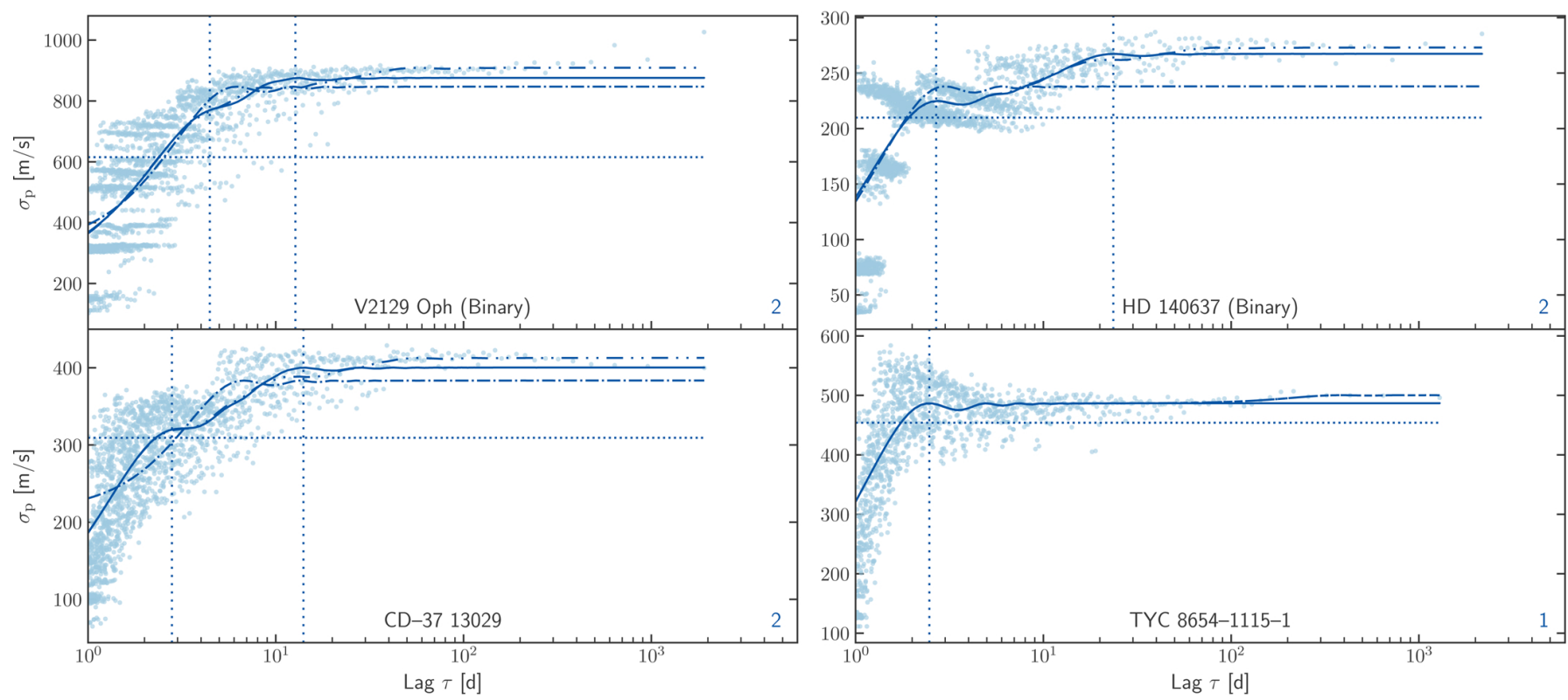

Fig. 2. Pooled results for the first 6 stars. Find plots for the remaining 21 stars in Fig. D.1. Blue symbols denote FEROS data and green symbols HARPS data. The lines show the best fits for the different numbers of sinusoidal signals fitted: dotted: zero signal (constant), dash-dotted: one signal, dashed: two signals, dash-dot-dotted: three signals. The solid line replaces the line which qualified as best fit using the F-test described in Sect. 3.4. The dotted vertical lines represent the periods of the identified signals and the number in the bottom right corner the number of identified signals. The model parameters are shown in Table 2. We note that $\sigma_{\mathrm{p}}$ and not $\sigma_{\mathrm{p}}^{2}$ is shown.

are used as the asymmetric error bars. An example of this distribution for HD 45184 is shown in Fig. E.1 (bottom left), where the lines indicate the best fits and confidence intervals. However, bootstrapping assumes the underlying data points to be mutually independent and well sampled. Since the statistical independence of the PVs is not fulfilled here and additional systematic errors might be present, the errors should be seen as lower limits to the real uncertainties.

\section{Results}

We derived an analytic activity-lag-function for the 27 stars (including the 7 binaries) that were selected by the criteria described in Sect. 2. The analytic function fitted to the PV data of each star is given by Eq. (4). This equation describes constant noise plus up to three independent sinusoidal signals as they would show up in the PVD in the case of infinite sampling. It has one free parameter for the constant plus two more for each signal identified. Those parameters represent the period and amplitude of the assumed underlying sinusoid. As it was integrated over the phase and we assume all phases to be covered roughly equally, no parameter for the phase or potential phase jumps is needed; see Appendix B. The results of the fits are presented in Table 2. Figure 2 shows those fits graphically as well as the PV for all stars and Fig. 3 then compares those to the age of the stars. With the exception of HD 51062 (highest dotted line), a clear spectroscopic binary (SB) as seen in the cross-correlation function (CCFs) returned by CERES, a clear correlation between age and RV scatter $\sigma$ is found. We excluded HD 51062 as well as the other six visual binaries from further analysis, and only use the six wide binaries as a consistency check with increased statistics below. As shown in Table 2, on average there were 1.6 individual signals with periods between 2.1 and 114 days identified for those 20 stars. These signals cause an increase of the stellar noise by roughly a factor of two when comparing the variance at the smallest lags with those at the largest lags, as can be seen in the
PVDs in Fig. 2. This means that if one is looking for planetary signals, not only does the amplitude of the signal of the planet $K_{\mathrm{pl}}$ decrease with $K_{\mathrm{pl}} \propto P^{-1 / 3}$, but also the underlying noise doubles when probing months instead of days.

In order to quantify this further and to make it possible for surveys to predict the amount of RV jitter before starting the observations, we fitted an empirical model, now adding a dependence on age to the model. Since the systematic errors are much larger than the formal errors on the curves, we did not account for those errors, or for the uncertainties of the ages. Instead, we reused the weights for each point derived earlier, but normalized them such that the sum of the weights equals one for each star. This procedure ensures that each star gets assigned the same weight, but still keeps the different weights for the individual points. The fitting is done using the python scipy.optimize.curve_fit least-square fitting routine, where we fit with all three parameters (age, lag, and standard deviation) in log space, decreasing the impact of extreme values. The errors are then determined using the square root of the diagonal entries of the covariance matrix of the fit results. This can be done since the weights are scaled such that the reduced $\chi^{2}$ equals unity. We used a shifted and stretched error function as our model. We chose the error function, since it asymptotically approaches different constant values in the positive and negative directions, similar to the PV signal of a sine wave; see Eq. (B.4). It is analytically described by

$\log \sigma(\hat{T}, \hat{\tau})=\kappa_{0} \cdot \hat{T}^{\kappa_{1}} \cdot\left[\operatorname{erf}\left(\omega_{0}+\hat{\tau} \cdot \omega_{1}\right)\right]-\epsilon$,

where $\hat{T}$ and $\hat{\tau}$ are the respective decadic logarithms of the stellar age $T$ in years and lag $\tau$ in days and $\sigma$ is returned in meters per second. $\kappa_{0}, \kappa_{1}, \omega_{0}, \omega_{1}$ and $\epsilon$ are free parameters of the model and erf is defined by $\operatorname{erf}(x):=\frac{1}{\sqrt{(\pi)}} \int_{-x}^{x} \mathrm{e}^{-t^{2}} \mathrm{~d} t$. The respective $\kappa_{i}$ and $\omega_{i}$ describe amplitude and angular frequency of the model, similar to $K$ and $2 \pi / P$ in the one-dimensional case given in Eq. (B.4). The parameter $\omega_{1}$ describes how steeply the noise $\sigma$ increases 


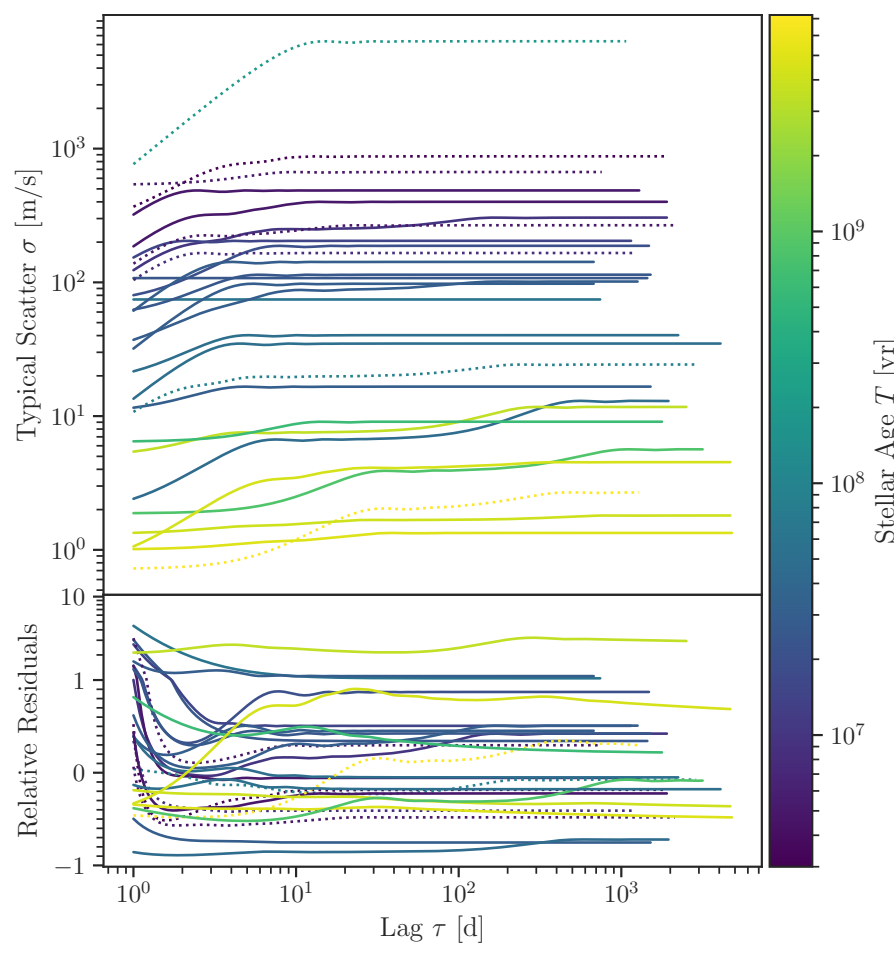

Fig. 3. Top: fitted RV jitter of the $28 \mathrm{RV}$ datasets of the 27 stars using the procedure described in Sect. 3.4. The color indicates the age of the star, showing a clear correspondence between RV jitter and age. The dotted lines corresponds to the binaries and are not used to fit the age-lagactivity model. The outlier at the top is HD 51062, the newly identified SB. Bottom: residuals of the above, ignoring HD 51062, from the more complex age-lag-activity model (b), divided by the model value. The model is described in Sect. 4, Eq. (6). We highlight the linear scale between -1 and 1 and a $\log$-scale for values greater than 1 . HD 51062 is excluded from the residuals.

with lag $\tau$, and $\epsilon$ is a simple offset since the error function goes through the origin. We call this model (a). The fitted parameter values and errors are given in Table 3 and the contours of the 2D function are plotted in Fig. 4 as white dotted lines. The most striking feature is the very strong dependence of the RV scatter on stellar age. Although this behavior was already known qualitatively, to the authors' knowledge the dependence is quantified for the first time here. In addition, the stellar noise increases when going to longer baselines: by a factor of 3.4 for stars with an age of $5 \mathrm{Myr}$ years, and 1.6 for stars with an age of $5 \mathrm{Gyr}$, when comparing a baseline of $10 \mathrm{yr}$ with a baseline of $1 \mathrm{~d}$. With this model, this increase happens on average such that $99 \%$ of the maximum activity is reached after approximately $10 \mathrm{~d}$. Thus, especially for young stars, the already very high noise level is increasing from $190 \mathrm{~m} \mathrm{~s}^{-1}$ for a lag of $1 \mathrm{~d}$ to $640 \mathrm{~m} \mathrm{~s}^{-1}$ for a lag of $10 \mathrm{~d}$ or longer.

Additionally, it would be interesting to know whether younger stars typically have longer or shorter periodic signals than older stars. To answer this, another free parameter $\delta$ was introduced, slightly changing Eq. (5) to

$\log \sigma(\hat{T}, \hat{\tau})=\kappa_{0} \cdot \hat{T}^{\kappa_{1}} \cdot\left[\operatorname{erf}\left(\omega_{0}+\frac{\hat{\tau} \cdot \omega_{1}}{\hat{T}^{\delta}}\right)\right]-\epsilon$,

which we now refer to as model (b). As shown in Table 3, we derive a value of $\delta=8.00 \pm 0.12$. The positive value of $\delta$ means that younger stars have shorter activity timescales than older ones, as can clearly be seen in the 2D function with solid white contours shown in Fig. 4. With this dependence, $99 \%$ of
Table 3. Least-square fit results of the simple model (a) from Eq. (5) and the slightly more complex model (b) described in Eq. (6) to the data of the 20 putatively single stars.

\begin{tabular}{lcccccc}
\hline \hline Mod. & $\kappa_{0}$ & $\kappa_{1}$ & $\omega_{0}$ & $\omega_{1}$ & $\delta$ & $\epsilon$ \\
\hline (a) & 454 & -2.463 & 1.077 & 1.164 & $\ldots$ & 1.382 \\
& \pm 31 & \pm 0.040 & \pm 0.007 & \pm 0.038 & $\ldots$ & \pm 0.041 \\
(b) & 312 & -2.294 & 1.015 & $12.8 \mathrm{M}$ & 8.00 & 1.261 \\
& \pm 20 & \pm 0.040 & \pm 0.008 & $\pm 3.7 \mathrm{M}$ & \pm 0.12 & \pm 0.041 \\
\hline
\end{tabular}

Notes. The first row gives the parameter values, the second the corresponding formal errors.

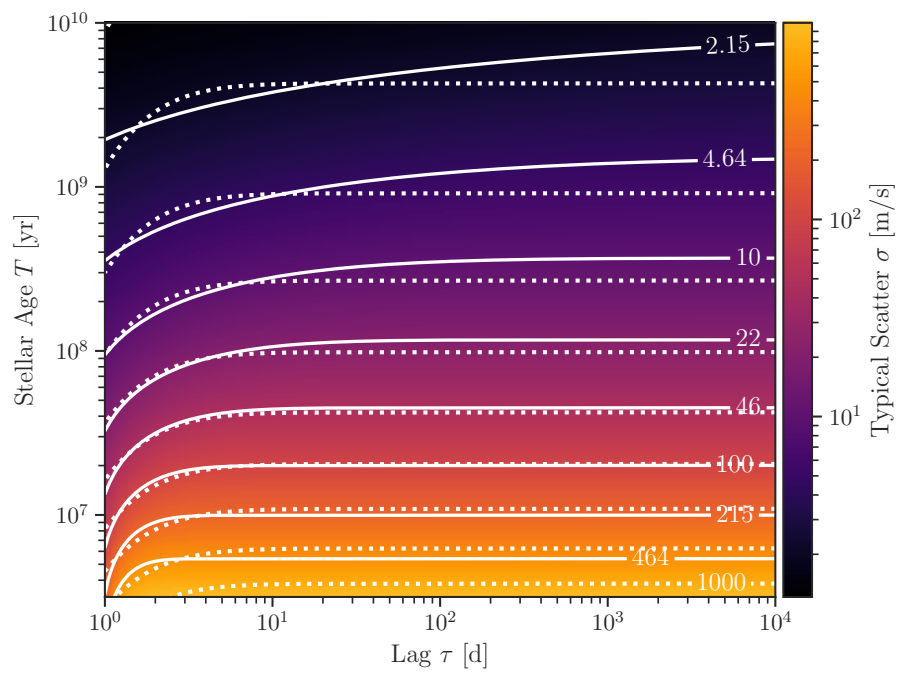

Fig. 4. Fitted activity model to the PV data excluding HD 51062. This model has been subtracted in Fig. 3. The color code and white solid lines with contour levels show the more complex model (b) of Eq. (6). As expected, the most important parameter is age. The increase of significant activity timescales with age can also be seen: $99 \%$ of the final activity is reached after $\sim 5 \mathrm{~d}$ for a 10 Myr-old star, but only after $\sim 30 \mathrm{yr}$ for a $10 \mathrm{Gyr}$-old star. The black dashed lines show the simpler model (a) of Eq. (5) where the timescales are forced to be the same for all ages.

the maximum activity will be reached after $2.5 \mathrm{~d}$ for stars with ages of $5 \mathrm{Myr}$ and after $14 \mathrm{~d}, 800 \mathrm{~d}$, and $10 \mathrm{yr}$ for stars with ages of $50 \mathrm{Myr}, 500 \mathrm{Myr}$, and $5 \mathrm{Gyr}$, respectively. The total increase with lag is a factor of 4 for 5 Myr-old stars and a factor of 1.8 for $5 \mathrm{Gyr}$-old stars. At the same time, the absolute jitter values for a lag of $10 \mathrm{~d}$ decrease from $516 \mathrm{~m} \mathrm{~s}^{-1}$ for a 5 Myr-old star to $41 \mathrm{~m} \mathrm{~s}^{-1}, 6.7 \mathrm{~m} \mathrm{~s}^{-1}$, and $1.9 \mathrm{~m} \mathrm{~s}^{-1}$ for stars with ages of $50 \mathrm{Myr}$, $500 \mathrm{Myr}$, and $5 \mathrm{Gyr}$, respectively.

\section{Discussion}

Without making use of any stellar models, we were able to determine the RV jitter as function of lag for 27 stars and to describe it with an analytic function for all of those using PV. However, since the assumption of statistical independence is violated in the pooled points, the F-test used to determine the number of sinusoidal signals identified is strictly speaking not applicable. Thus, even though the results appear convincing, one cannot put numbers on the significance of an identified signal, one of the original plans to characterize stars even further. Consequently, the errors determined using bootstrapping need to be considered as lower limits because of this lack of statistical independence. 


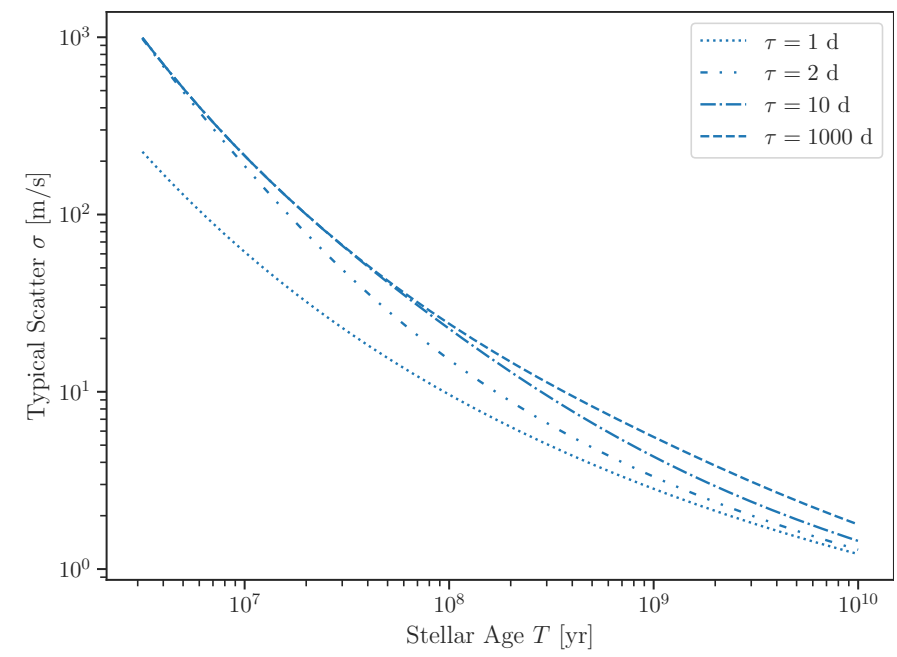

Fig. 5. Four slices for different lags through the activity model (b) presented in Fig. 4 with solid lines. The slices show the strong dependance on age, but also on lag, in particular for the older stars.

For the future, a MC simulation on the original data with scaled error bars would perhaps return more realistic errors. But since we neither make use of the number of signals identified nor of the errors, we did not pursue this further.

This lack of statistical independence has the largest influence when little and clustered data is present. This is often the case in the HARPS data taken after the intervention, resulting in a horizontal line of pooled data, as seen for example in HD 30495 in Fig. D.1. This is because often only a few or even one "box" has at least two data points, allowing calculation of the variance. In the case of HD 30495, multiple data points were taken in one night, with the shortest time period between two nights of data capture being $44 \mathrm{~d}$. Thus, the PV only changes for a lag of $44 \mathrm{~d}$ or larger, sometimes resulting in a jump in the PVD. Fortunately, fewer data points are used in these cases, down-weighting the influence of these cases. Also, the selection criteria presented in Sect. 2 try to limit the occurrence of these phenomena.

For stellar activity there are three main characteristic timescales involved (e.g., Borgniet et al. 2015): first, the stellar rotation causing rotational modulation of active regions and their effects with typical timescales of days to a few weeks. Second, the reconfiguration of active regions with typical timescales of several weeks. And third, the stellar activity cycle with typical timescales of several years or decades. Of those three, the second is the least periodic and has a rather unsharp timescale; furthermore, it overlaps with the stellar rotation timescale, meaning that the two are difficult to disentangle; see for example Giles et al (2017). Many stars show an increase of the RV jitter up to a few days or weeks followed by a plateau. This is probably due to the stellar rotation and reconfiguration of the active regions. In addition, especially the older, more quiet stars show another strong increase with a lag of a few years. This is probably related to the stellar activity cycle. However, since this increase is often close to the probed baseline, we might often only probe a part of this additional noise term.

For very active stars, the white noise term $A$ of Eq. (4) is often consistent with zero, taking the error bars into account. This probably means that the periodic signal dominates the fit and therefore $A$ cannot be determined very well numerically instead of truly being zero.

Since the ages of all stars are known, we could determine an empirical but still analytic model of the age-lag-activity relation based on the 20 presumably single stars. This model shows that the typical RV jitter of a star depends strongly on the age of the star, ranging from $516 \mathrm{~m} \mathrm{~s}^{-1}$ for a $5 \mathrm{Myr}$-old star down to $2.5 \mathrm{~m} \mathrm{~s}^{-1}$ for a $5 \mathrm{Gyr}$-old star - when probing lags of $10 \mathrm{yr}$. When considering a timescale of $1 \mathrm{~d}$ only, the RV jitter is smaller by roughly a factor of 4 for stars with ages of $5 \mathrm{Myr}$ and a factor of 1.7 for stars with an age of 5 Gyr. The rate by which this jitter then increases with lag strongly depends on the age of the stars, where according to the model younger stars reach their maximum in a few days and older stars take up to thousands of years. We note that the maximum baseline of our measurements is about $13 \mathrm{yr}$, meaning that we cannot say anything about the contribution of activity cycles that operate on even longer timescales. The ages of the single stars probed reach from $5 \mathrm{Myr}$ to 5 Gyr and cover spectral types from F6 to M0; see Table 1 and Fig. 1. By construction, each star is assigned the same weight, and since 14 of the 20 stars are younger than $100 \mathrm{Myr}$, the result is skewed towards relatively young stars and thus is also most robust for those young stars. We also note that the individual scatter of $\sigma_{\mathrm{p}}$ of the stars is about a factor of two, as can be seen in the residuals of Fig. 4. This might hint towards another dependence of the activity on spectral type or metallicity. However, more data are needed to analyze this dependence.

To test our model with increased statistics, we derived the age-lag relationship of model (b) with the six additional wide ( $\geq 27 \mathrm{AU}$ projected separations) binary systems, that is, all stars except the SB HD 51062. Since the activity of these stars is on average slightly lower than that of the similar-aged single stars (see Fig. 3) the RV data for these stars are likely not dominated by the binary companions, justifying this approach. The derived values are $\kappa_{0}=98.4 \pm 4.1, \kappa_{1}=-1.544 \pm 0.029, \omega_{0}=$ $1.1612 \pm 0.0071, \omega_{1}=(3.17 \pm 1.56) \times 10^{6}, \delta=7.36 \pm 0.25$ and $\epsilon=2.471 \pm 0.066 \mathrm{~m} \mathrm{~s}^{-1}$. Despite individual values changing more than the formal errors (e.g., $\kappa_{0}$ from 312 to 98 ), the typical scatter $\sigma$ does not change by more than $20 \%$ in the probed parameter space (ages from $5 \mathrm{Myr}-10 \mathrm{Gyr}$, lags from $1 \mathrm{~d}$ $-10^{4} \mathrm{~d}$ ). Also the trend of longer activity timescales for older stars, quantified by the $\delta$ parameters, remains of the same order and clearly positive. The major change is a decrease of the RV scatter by slightly less than $20 \%$ for 5 Myr-old stars and by $10 \%$ for 5 Gyr-old stars. It remains the same within a few percent for stars of intermediate ages $(\sim 50 \mathrm{Myr}-1 \mathrm{Gyr})$. Therefore, this test strengthens our confidence in the robustness of our model.

This means that for RV exoplanet-hunting surveys using state of the art instruments for example, one should be aware that for stars younger than a few hundred million years the limit is set by the stellar jitter and not by instrumental precision and the age is a crucial parameter. For example, the RV SPY survey excludes stars younger than about $5 \mathrm{Myr}$ although its goal is to find planets around young stars. Additionally, RV SPY focuses on searching for hot Jupiters, where the stellar jitter is slightly smaller and the signal of the planet is larger than for longer periodic planets (Zakhozhay et al., in prep.).

\section{Conclusions}

Here, we show that the PV method can be used to model and determine stellar activity timescales and amplitudes. We applied PV to 28 data sets of 27 different stars. We find an empirical relation between RV scatter, stellar age, and lag $\tau$. We found a very strong dependence of RV jitter on age. Further, the RV scatter roughly doubles with the lag $\tau$ when probing over timescales of months instead of a few days. 
This relation is not only important for stellar modeling, but also for developing an observing strategy for RV exoplanet surveys, especially if young stars are involved, such as predicting the RV jitter for young K/G dwarfs common in the TESS survey. Also, in searches for hot Jupiters, dense sampling is approximately twice as sensitive as long-term random sampling. One survey making use of the findings in this paper is the RV SPY survey with FEROS, looking for hot Jupiters around young stars (Zakhozhay et al., in prep.).

Acknowledgements. This research has made use of the NASA Exoplanet Archive, which is operated by the California Institute of Technology, under contract with the National Aeronautics and Space Administration under the Exoplanet Exploration Program. This research made use of Astropy (http://www . astropy.org), a community-developed core Python package for Astronomy (Astropy Collaboration 2013, 2018). This research has made use of the SIMBAD database, operated at CDS, Strasbourg, France Thanks also to Diana Kossakowski for fruitful discussions about Gaussian processes and its limitations on this application and to Peter Markowski for his help on statistical issues. We would also like to thank the anonymous referee for his or her helpful comments and ideas, which helped to improve the paper.

\section{References}

Astropy Collaboration (Robitaille, T. P., et al.) 2013, A\&A, 558, A33 Astropy Collaboration (Price-Whelan, A. M., et al.) 2018, AJ, 156, 123

Borgniet, S., Meunier, N., \& Lagrange, A.-M. 2015, A\&A, 581, A133

Brahm, R., Jordán, A., \& Espinoza, N. 2017, PASP, 129, 034002

Brown, L. D., \& Levine, M. 2007, Ann. Stat., 35, 2219

Chen, C. H., Mittal, T., Kuchner, M., et al. 2014, ApJS, 211, 25

David, T. J., Hillenbrand, L. A., Petigura, E. A., et al. 2016, Nature, 534, 658

Desidera, S., Covino, E., Messina, S., et al. 2015, A\&A, 573, A126

Dobson, A. K., Donahue, R. A., Radick, R. R., \& Kadlec, K. L. 1990, in Cool Stars, Stellar Systems, and the Sun, ed. G. Wallerstein, ASP Conf. Ser., 9, 132

Donahue, R. A., \& Dobson, A. K. 1996, in Cool Stars, Stellar Systems, and the Sun, eds. R. Pallavicini, \& A. K. Dupree, ASP Conf. Ser., 109, 599

Donahue, R. A., Dobson, A. K., \& Baliunas, S. L. 1995, in American Astronomical Society Meeting Abstracts \#186, BAAS, 27, 843

Donahue, R. A., Dobson, A. K., \& Baliunas, S. L. 1997a, Sol. Phys., 171, 191

Donahue, R. A., Dobson, A. K., \& Baliunas, S. L. 1997b, Sol. Phys., 171, 211
Donati, J. F., Moutou, C., Malo, L., et al. 2016, Nature, 534, 662 Dumusque, X. 2018, A\&A, 620, A47

Ercolano, B., \& Pascucci, I. 2017, R. Soc. Open Sci., 4, 170114

Eyer, L., \& Genton, M. G. 1999, A\&AS, 136, 421

Foreman-Mackey, D. 2016, https://doi .org/10.5281/zenodo. 45906

Giles, H. A. C., Collier Cameron, A., \& Haywood, R. D. 2017, MNRAS, 472, 1618

Hughes, P. A., Aller, H. D., \& Aller, M. F. 1992, ApJ, 396, 469

Ibukiyama, A., \& Arimoto, N. 2002, A\&A, 394, 927

Kaufer, A., Stahl, O., Tubbesing, S., et al. 1999, The Messenger, 95, 8

Kürster, M., Endl, M., Els, S., et al. 2004, in Planetary Systems in the Universe, ed. A. Penny, IAU Symp., 202, 36

Lachaume, R., Dominik, C., Lanz, T., \& Habing, H. J. 1999, A\&A, 348, 897

Lannier, J., Delorme, P., Lagrange, A. M., et al. 2016, A\&A, 596, A83

Lenzen, R., Hartung, M., Brandner, W., et al. 2003, in Instrument Design and Performance for Optical/Infrared Ground-based Telescopes, eds. M. Iye, \& A. F. M. Moorwood, Proc. SPIE, 4841, 944

Lindegren, L., \& Dravins, D. 2003, A\&A, 401, 1185

Lo Curto, G., Pepe, F., Avila, G., et al. 2015, The Messenger, 162, 9

Maldonado, J., Martínez-Arnáiz, R. M., Eiroa, C., Montes, D., \& Montesinos, B. 2010, A\&A, 521, A12

Mason, B. D., Wycoff, G. L., Hartkopf, W. I., Douglass, G. G., \& Worley, C. E. 2001, AJ, 122, 3466

Mayor, M., Pepe, F., Queloz, D., et al. 2003, The Messenger, 114, 20

Meunier, N., \& Lagrange, A.-M. 2019, A\&A, 628, A125

Mohler-Fischer, M. 2013, PhD Thesis, University of Heidelberg, Germany

Pourbaix, D., Tokovinin, A. A., Batten, A. H., et al. 2004, A\&A, 424, 727

Rawlings, J. O., Pantula, S. G., \& Dickey, D. A. 1998, in Applied Regression Analysis, 2nd edn. (Berlin: Springer), Springer Texts Stat., XVIII, 657 S

Rousseeuw, P. J., \& Croux, C. 1993, J. Am. Stat. Assoc., 88, 1273

Rousset, G., Lacombe, F., Puget, P., et al. 2003, in Adaptive Optical System Technologies II, eds. P. L. Wizinowich, \& D. Bonaccini, Proc. SPIE, 4839, 140

Squires, G. L. 2001, Practical Physics, 4th edn. (Cambridge: Cambridge University Press), XI, $212 \mathrm{~S}$

Stone, J. M., Skemer, A. J., Hinz, P. M., et al. 2018, AJ, 156, 286

Tamura, M. 2016, Proc. Jpn. Acad. Ser. B, 92, 45

Tucci Maia, M., Ramírez, I., Meléndez, J., et al. 2016, A\&A, 590, A32

Vican, L. 2012, AJ, 143, 135

Weise, P. 2010, PhD Thesis, University of Heidelberg, Germany

Weise, P., Launhardt, R., Setiawan, J., \& Henning, T. 2010, A\&A, 517, A88

Yu, L., Donati, J.-F., Hébrard, E. M., et al. 2017, MNRAS, 467, 1342

Zechmeister, M., Reiners, A., Amado, P. J., et al. 2018, A\&A, 609, A12 


\section{Appendix A: Analytic description of the PV}

The variance of a data set $y_{i}=1, \ldots, n$ is given by

$\sigma^{2}(n)=\frac{1}{n-1} \sum_{i=1}^{n}\left(y_{i}-\bar{y}\right)^{2}$,

where $\bar{y}=\frac{1}{n} \sum_{i=1}^{n} y_{i}$. For sufficiently large $n$, we have

$\sigma^{2} \approx \frac{1}{n} \sum_{i=1}^{n}\left(y_{i}-\bar{y}\right)^{2}=\overline{y^{2}}-\bar{y}^{2}$,

where $\overline{y^{2}}=\frac{1}{n} \sum_{i=1}^{n} y_{i}^{2}$.

In the limit of equidistant and infinitely dense sampling of the data, we can replace the sums by integrals; Eq. (A.2) then becomes

$\sigma^{2}(\tau)=\frac{1}{\tau} \int_{0}^{\tau} y^{2}(t) \mathrm{d} t-\left(\frac{1}{\tau} \int_{0}^{\tau} y(t) \mathrm{d} t\right)^{2}$,

where $\tau$ is the timescale over which the variance is to be evaluated.

As we see below, for periodic functions the shape of $\sigma^{2}(\tau)$ depends strongly on the phase of the periodic function. In practice however, the phase of a signal is often sampled repeatedly in a random fashion, thus also averaging over potential variations in the phase. Therefore, we consider the phase-averaged variance, with $\delta \in[0,2 \pi)$ the phase of the signal $y(\delta)$

$\overline{\sigma^{2}}(\tau)=\frac{1}{2 \pi} \int_{0}^{2 \pi} \sigma^{2}(\tau, \delta) \mathrm{d} \delta$.

We note that we take averages over the signal $\bar{y}$ with respect to the selected time scale $\tau$, whereas we take the average over the variance $\overline{\sigma^{2}}$ with respect to the phase of the signal $\delta$.
In the analytic case (infinitely dense sampling) the PV of a data set is given by inserting Eq. (A.3) into Eq. (A.4). In reality one might see effects of transient oscillations if the signal is not sampled densely at all phases. To minimize the influence of those effects, the selection criteria for the sampling described in Sect. 2 were applied.

\section{Appendix B: Analytic PV of sinusoids}

As an example we consider the PV of a sine wave:

$y(t)=K \sin \left(\frac{2 \pi}{P} t-\delta\right)$,

where $K$ is the (semi-) amplitude, $P$ is the period, and $\delta \in[0,2 \pi)$ the phase.

Evaluating Eq. (A.3) for the sine function of Eq. (B.1) yields the scaled variance

$$
\begin{aligned}
\Omega^{2}(\theta)= & \frac{1}{2}-\frac{\sin (2 \theta-2 \delta)+\sin (2 \delta)}{4 \theta} \\
& -\left[\frac{\cos \delta-\cos (\theta-\delta)}{\theta}\right]^{2},
\end{aligned}
$$

where $\Omega^{2}:=\sigma^{2} / K^{2}$ and $\theta=2 \pi \tau / P$. Inserting Eq. (B.2) into Eq. (A.4) yields the normalized, analytic PV of a sine wave

$\overline{\Omega^{2}}(\theta)=\frac{1}{2}+\frac{\cos \theta-1}{\theta^{2}}$,

or

$\overline{\sigma^{2}}(\tau)=K^{2}\left[\frac{1}{2}+\frac{\cos (2 \pi \tau / P)-1}{(2 \pi \tau / P)^{2}}\right]$.

We have $\lim _{\tau \rightarrow 0} \overline{\sigma^{2}}(\tau)=0$ and $\overline{\sigma^{2}}(\tau)=K^{2} / 2$ for $\tau=P$ and also for $\tau \gg P$. 


\section{Appendix C: Radial-velocity data}
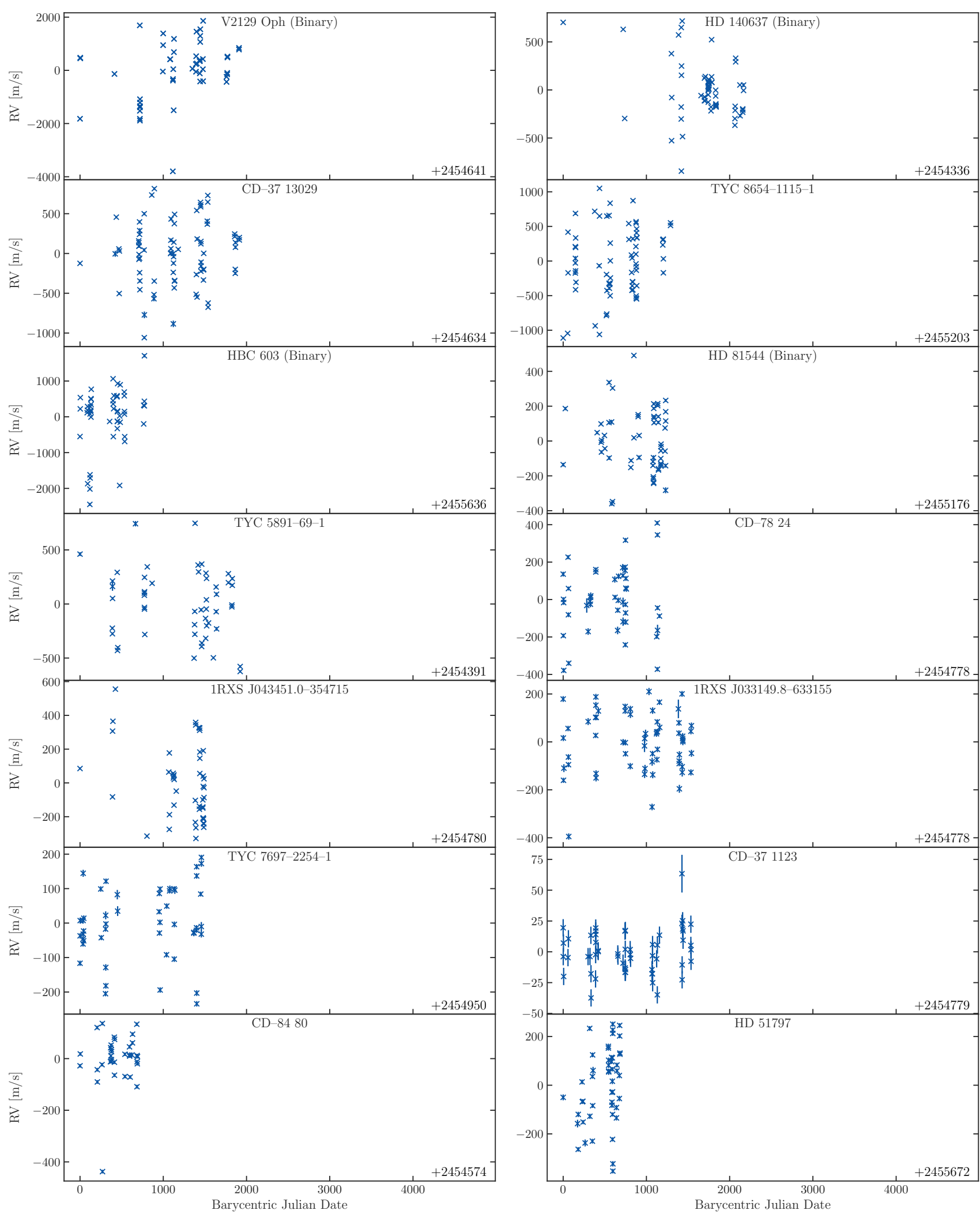

Fig. C.1. Radial velocity data for all 27 stars after removing the bad data as described in Sect. 2 and subtracting the mean of each data set. Green symbols denote HARPS and blue symbols FEROS data. The offset of the Julian Date on the $x$-axis is given in the bottom right of each plot. The green vertical lines mark the fiber change of HARPS where the data sets were split. One can see how some data show clear jumps there while others do not. Jumps in the PVD in Fig. 2 as in HD 51062 or HD 25457 can be explained by clustered (e.g., HD 25457) or relatively sparse (e.g., HD 51062) data. 
A\&A 632, A37 (2019)

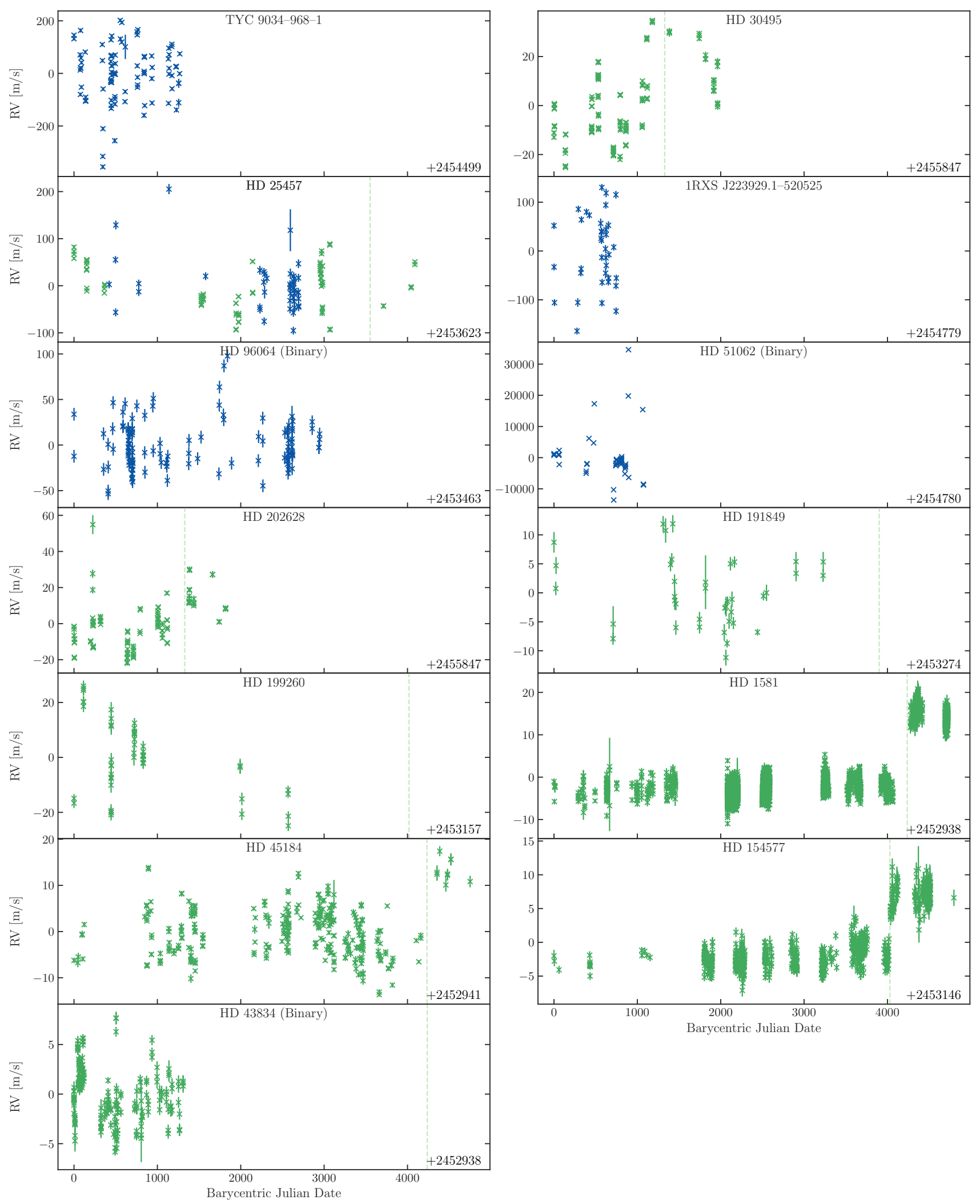

Fig. C.1. continued. 
S. S. Brems et al.: Radial-velocity jitter of stars as a function of observational timescale and stellar age

\section{Appendix D: Pooled variance diagrams}
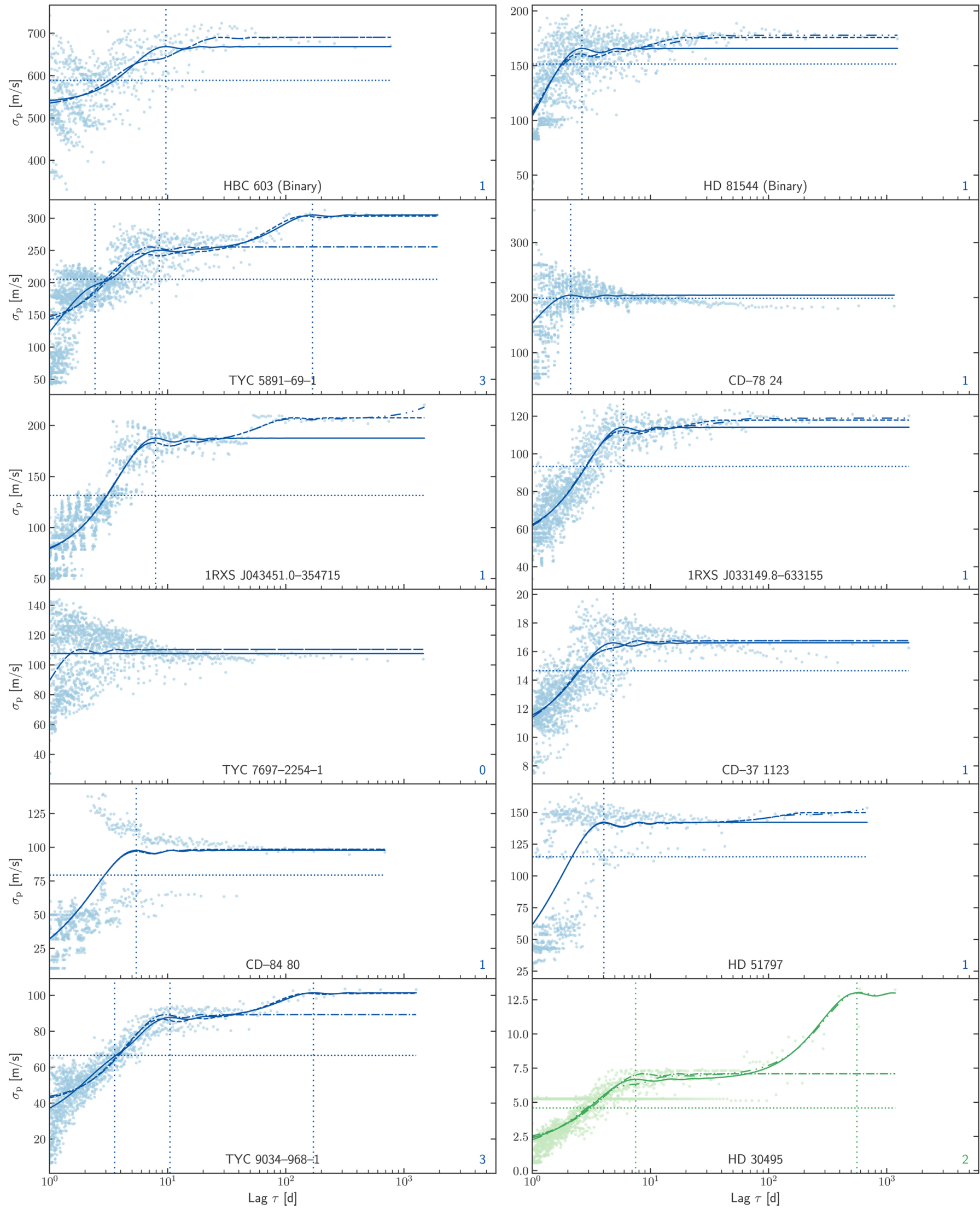

Fig. D.1. Continued from Fig. 2. 
A\&A 632, A37 (2019)
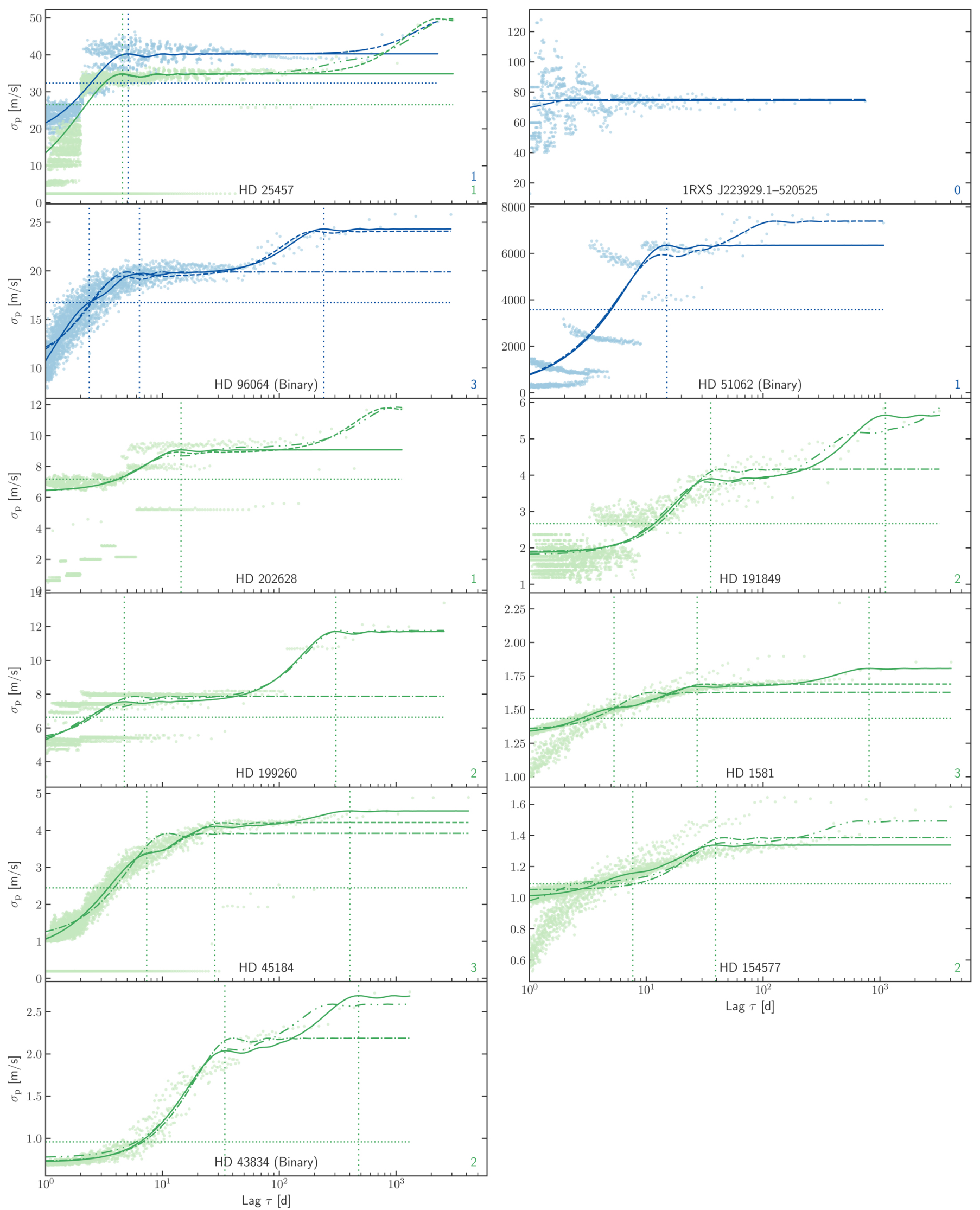

Fig. D.1. continued. 


\section{Appendix E: Pooled variance error estimate}

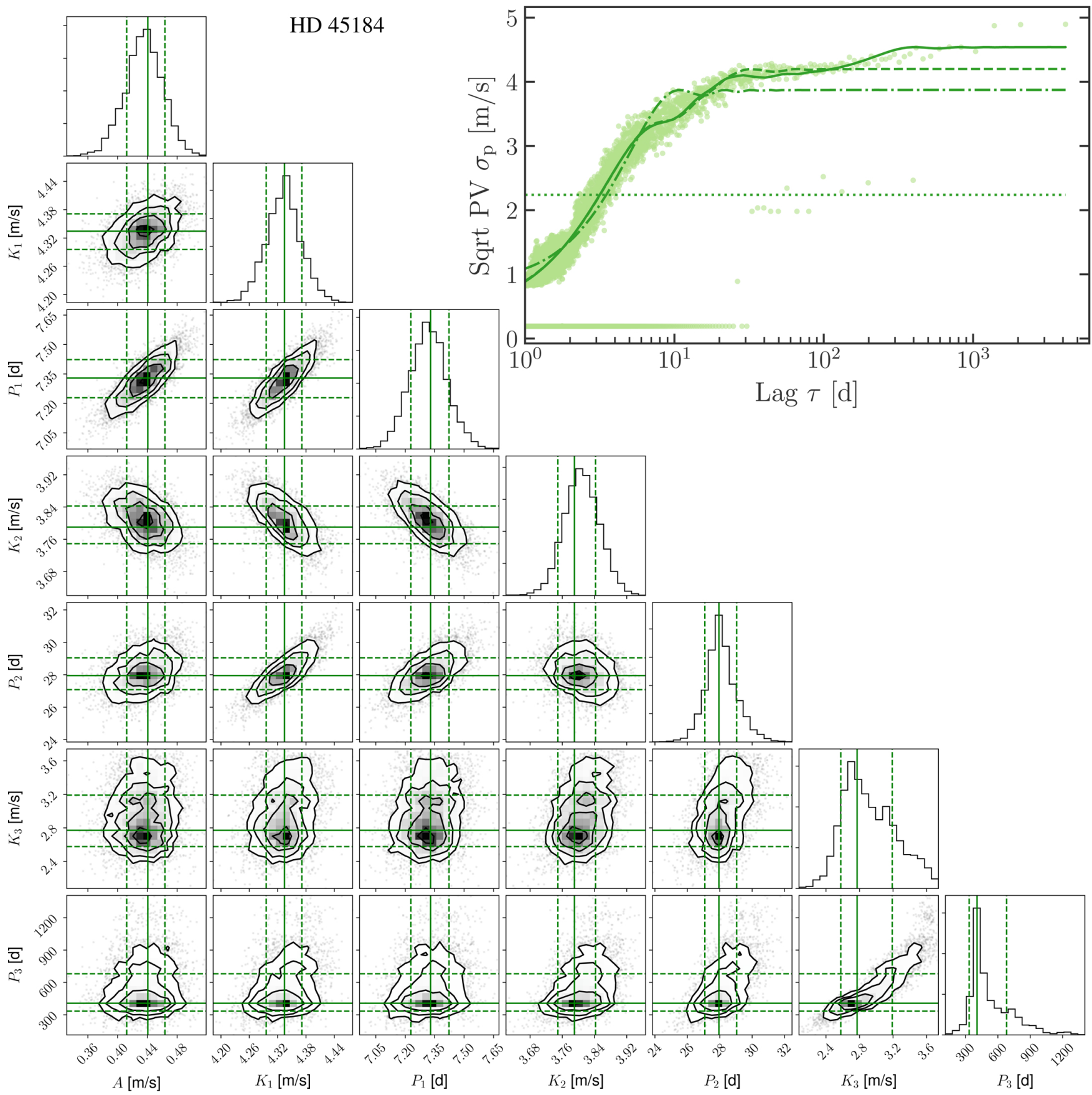

Fig. E.1. Bottom left: results of the bootstrapping procedure shown as an example for HD 45184. Shown is a corner plot using the Foreman-Mackey (2016) python package. A denotes the offset where as $K_{i}$ and $P_{i}$ show the amplitudes and periods of the $i$ th signal, as defined in Eq. (4). The solid green bars denote the fit to the original data, whereas the dashed lines denote the $1 \sigma$-confidence levels. In the case of HD 45184 , three signals were identified as significant by the F-test, which are shown here. Top right: PV plot: the points mark the results of the PV and the curves the fits with different numbers of sinusoidal signals modeled: dotted: zero (constant), dash-dotted: one sinusoid, dashed: two sinusoids, solid: three sinusoids (best fit). The flat line of points at the bottom are due to the sparse sampling after the HARPS intervention, where only two times two observations are taken within less than 20 days, and those were respectively taken on the same nights; see Fig. C.1. 